\title{
Europe's X-ray observatory defies the jinx...
}

Munich

The jinx that destroyed or limited a string of $\mathrm{X}$-ray-astronomy missions launched in the past nine months does not seem to have affected the European Space Agency's (ESA's) new XMM-Newton X-ray observatory. The first images, presented last week, show that the observatory's three X-ray cameras, its optical monitor and its two spectroscopes are operating well.

ESA also announced that it had added the name Newton to the observatory's working name of XMM (X-ray Multi-Mirror), in honour of Isaac Newton, who created the theory of gravity and discovered the Sun's spectrum.

$\mathrm{X}$-ray astronomers are pleased that Newton, which was launched two months ago, has avoided the damage suffered by a similar mission, the US space agency NASA's Chandra. Low-energy protons partly damaged Chandra's cameras a few weeks after launch last July (see Nature 401, 415; 1999).

The Newton observatory comprises three telescopes, each consisting of a nest of $58 \mathrm{mir}$ rors, which are designed to detect $\mathrm{X}$-ray photons from deep space. X-rays are emitted by very hot matter, so Newton can observe very hot and violent events in the cosmos, allowing studies of the evolution and death of stars, the structure and nature of the interstellar medium, and the physics of galaxies and quasars.

In addition to the damage to Chandra, Xray astronomers had previously witnessed the loss of Abrixas, a small German-built Xray satellite, immediately after its launch last May (see Nature 399, 93; 1999). Last week also saw the loss of Japan's major X-ray mission Astro-E, when its launcher failed to push it into the correct orbit (see right).

Newton scientists say they could have been affected by the problem that struck Chandra, because, like their US colleagues, they had not predicted the damaging effects of one type of cosmic radiation in the deepspace orbit chosen to avoid the influence of the Earth's atmosphere and used by both Chandra and Newton.

Damaging low-energy protons in the Van Allen belts through which both vehicles fly were funnelled by the telescope mirrors onto the cameras. Eight of the ten sensors in Chandra's cameras are 'front-illuminated', making them vulnerable to damage. "Normally these protons are stopped, but obviously they are reflected off the mirrors and we did not anticipate this," says Richard Mushotzky, an astrophysicist at the Goddard Space Flight Center near Washington, and a member of Chandra's science working group.

The damage to Chandra's cameras does not affect the image resolution. But the spectral resolution, which allows the chemical composition of the observed objects to be
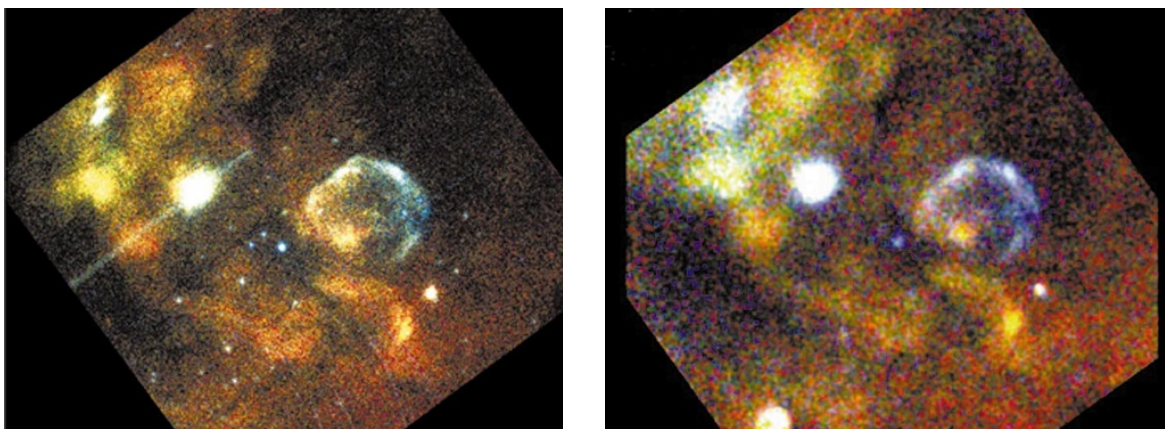

In focus: the picture on the left shows one of the first images taken by XMM-Newton, the Tarantula Nebula, part of the Large Magellanic Cloud. The one on the right shows the same region taken previously by ROSAT, a German X-ray satellite that went out of operation over a year ago.

analysed, has been reduced by up to a factor of five. In addition, the mission's ability to analyse X-ray sources that are greater over a field of view than one-fifth of the size of the full moon is also compromised, and these can now be observed, less efficiently, only with the undamaged 'back-illuminated' sensors.

Newton scientists say they have learnt from Chandra's misfortune. Having been more cautious with the original design of their EPIC X-ray cameras, which also include damage-sensitive front-illuminated sensors, they had incorporated a moving shield to allow the cameras to be shut off in the event of any unpredicted radiation damage while passing through the Van Allen belts or during a sunstorm, despite the extra resources this required.
Newton will make up for Chandra's partial loss, as the three big missions were originally complementary, so the weaknesses of one mission could be made up for by strengths in the others. Newton was optimized for sensitivity and for medium-tohigh-resolution spectroscopy, Chandra for angular resolution - which has not been affected - and Astro-E for very-high-resolution spectroscopy.

"We are lucky that Newton is well suited to looking at large diffuse sources such as clusters of galaxies, just the capability we lost," says Claude Canizares, an astrophysicist from the Massachusetts Institute of Technology and principal investigator of a Chandra instrument. "But we have completely lost the important piece of the scientific pie that Astro-E would have offered." Alison Abbott

\section{.. . but Astro-E is yet another Japanese failure}

Tokyo

X-ray astronomers in Japan received a major setback last week with the loss of Astro-E, a joint US-Japanese satellite carrying several innovative X-ray sensors. The failure is the latest in a series of accidents, and could have serious repercussions for Japan's space policy.

The satellite missed its orbit after an engine failure in the first stage of its launch vehicle, an M-V rocket built at Japan's second space agency, the Institute of Space and Astronautical Science (ISAS).

In addition to four X-ray imaging spectrometers, Astro-E carried a novel X-ray spectrometer - the fruit of collaboration between the US space agency NASA's Goddard
Space Flight Center and ISAS that was to have measured the heat created by $\mathrm{X}$-rays rather than converting them into electrical charges, and a 'hard' X-ray detector, built jointly by scientists at ISAS and the University of Tokyo.

For Kazuo Makishima, a professor at the High Energy Astrophysics Laboratory at the University of Tokyo, who was involved in building the hard $\mathrm{X}$-ray detector, the loss of Astro-E is a serious blow to $\mathrm{X}$-ray astronomy in Japan.

Yasunori Matogawa of ISAS, the key person behind Japan's Xray satellite programme, says the failure has come at a "a very bad moment" for Japan. "With XMMNewton in Europe and Chandra in the [United States], we were looking towards a period of exciting research and competition."

Japan should strive to launch a satellite similar to Astro-E as soon as possible, rather than trying to produce improved detectors, argues Matogawa. But other launch commitments make it unlikely that a new mission could take place before 2004 .

Following last week's failure, leading Japanese politicians have repeated calls for the country's two space agencies, ISAS and the National Space Development Agency (NASDA), to be merged into a single organization.

Such a move was suggested a few months ago, when one of NASDA's H-II rockets was destroyed because of an engine failure.

Robert Triendl 